
inhibition factor in drug allergy. Study 2009-2014

\title{
Pruebas modificadas de degranulación de basófilos y factor de inhibición de migración de leucocitos en alergia a fármacos. Estudio de 2009 a 2014
}

\author{
María de Lourdes Irigoyen-Coria, ${ }^{1}$ María Isabel Rojo-Gutiérrez, ${ }^{2}$ Rubén Humberto Meyer-Gómez, ${ }^{3}$ \\ Isabel Leyva-Carmona, ${ }^{4}$ Víctor Manuel Zendejas-Buitrón, ${ }^{5}$ Angélica Dinorah García-Ruiz, ${ }^{5}$ \\ Jaime Mellado-Ábrego, ${ }^{2}$ Ilayalid García-Ortega, ${ }^{5}$ Gloria Castillo-Narváez ${ }^{2}$
}

\begin{abstract}
Background: Adverse reactions to drugs are increasing and there are few studies for the diagnosis. Objective: To determine the utility of modified basophil degranulation (MBD) test and modified leukocyte migration inhibition factor (MLMIF) test to prove drug hypersensitivity.

Methods: 177 patients of both sexes were studied with the diagnosis of drug hypersensitivity, determining MBD, MLMIF, or both, between 2009 and 2014. They were matched with positive and negative controls and the non-allergic population. Applications are issued according to the type of hypersensitivity, considering type I MBD and type IV MLMIF.

Results: 170 patients (96.04\%) were positive to at least one drug (RR = 4.71). $561 \mathrm{MBD}(73.62 \%)$ and 201 MLMIF (26.37\%) were performed. Female sex was more frequent $(64.41 \%)$; the average age was 38.5 . MBD was positive in $70.23 \%$ and MLMIF in $67.16 \%$. The test sensitivity was increased complementarily and with two dilutions. The correlation of MBD and MLMIF was positive and highly significant.

Conclusions: Women have more drug reactions. Modified MBD test is useful at any age. Since medications can activate one or other hypersensitivity mechanism, it is important to request the tests simultaneously.
\end{abstract}

Keywords: Hypersensitivity; Allergy; Adverse reactions to drugs; Drugs

Este artículo debe citarse como: Irigoyen-Coria ML, Rojo-Gutiérrez MI, Meyer-Gómez RH, Leyva-Carmona I, Zendejas-Buitrón VM, García-Ruiz AD, Mellado-Ábrego J, García-Ortega I, Castillo-Narváez G. Pruebas modificadas de degranulación de basófilos y de factor de inhibición de la migración de leucocitos en población con alergia a fármacos. Estudio de 2009 a 2014. Rev Alerg Mex. 2016;63(4):342-350

${ }^{1}$ Laboratorios Clínicos Especializados Integrales Lindavista. Ciudad de México, México

${ }^{2}$ Secretaría de Salud, Hospital Juárez de México, Servicio de Alergia e Inmunología. Ciudad de México, México

${ }^{3}$ Hospital Ángeles del Pedregal. Ciudad de México, México ${ }^{4}$ Instituto de Seguridad y Servicios Sociales de los Trabajadores del Estado, Hospital Regional $1^{\circ}$ de Octubre. Ciudad de México, México

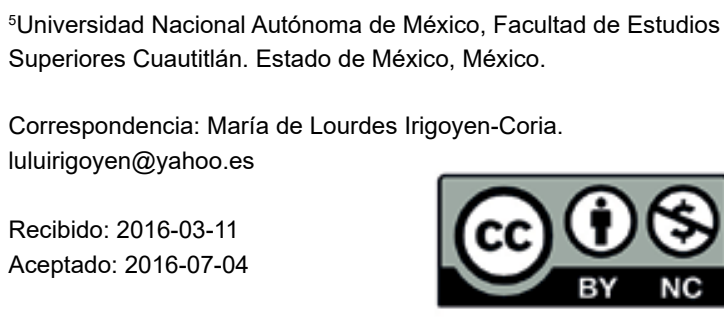

${ }^{5}$ Universidad Nacional Autónoma de México, Facultad de Estudios

Correspondencia: María de Lourdes Irigoyen-Coria. luluirigoyen@yahoo.es

Recibido: 2016-03-11

do: 2016-07-04 


\section{Resumen}

Antecedentes: Existe incremento de reacciones adversas a medicamentos y pocos estudios para el diagnóstico.

Objetivo: Determinar la utilidad de pruebas modificadas de degranulación de basófilos (DB) y del factor inhibidor de la migración de leucocitos (LIF, leukocyte migration inhibition factor) para comprobar la hipersensibilidad a medicamentos.

Métodos: Se estudiaron 177 pacientes, de uno y otro sexo, con diagnóstico de hipersensibilidad a medicamentos, en quienes se determinó pruebas modificadas de DB, LIF, o ambas entre 2009 y 2014. Se parearon con controles positivos, negativos y población no alérgica. Las solicitudes se emitieron de acuerdo con el tipo de hipersensibilidad, considerando tipo I a DB y tipo IV a LIF Resultados: 170 pacientes (96.04\%) fueron positivos al menos a un medicamento (RR, 4.71). Se realizaron 561 pruebas modificadas de DB (73.62\%) y 201 de LIF (26.37\%). El sexo femenino fue más frecuente $(64.41 \%)$; la edad promedio fue de 38.5 años. La prueba modificada de DB resultó positiva en $70.23 \%$ y la de LIF en $67.16 \%$. La sensibilidad de las pruebas se incrementó en forma complementaria y a dos diluciones. La correlación de las pruebas fue altamente significativa.

Conclusiones: Las mujeres presentan más reacciones a fármacos. La prueba modificada de DB es útil en cualquier edad. Como los medicamentos pueden activar uno u otro mecanismo de hipersensibilidad es importante solicitar las pruebas simultáneamente.

Palabras clave: Hipersensibilidad; Alergia; Reacciones adversas a medicamentos; Fármacos

\author{
Abreviaturas y siglas \\ CPA, células presentadoras de antígenos \\ $\mathrm{DB}$, degranulación de basófilos \\ INF-y, interferón gamma \\ LIF, leukocyte migration inhibiton factor \\ $\mathrm{MHC}$, complejo mayor de histocompatibilidad
}

NK, células natural killer

RAM, reacciones adversas a medicamentos

$\mathrm{RR}$, riesgo relativo

TCR, receptor de células T

TNF- $\alpha$, factor de necrosis tumoral alfa

\section{Antecedentes}

El aumento en el uso, abuso, variedad y complejidad química de los medicamentos aunado a la autoprescripción ha incrementado la incidencia de reacciones adversas a medicamentos (RAM). Establecer con certeza el diagnóstico de reacción alérgica causada por medicamentos es difícil debido a que los fármacos tienen la capacidad de activar uno o más mecanismos de hipersensibilidad, por lo que las RAM se consideran un problema de salud pública por su alta mortalidad, morbilidad y costos. ${ }^{1,2}$

El principal órgano de choque en las RAM es la piel y entre los fármacos implicados están los antibióticos (penicilinas y sulfas), analgésicos, antiinflamatorios no esteroideos (AINE) y los que actúan en el sistema nervioso central. ${ }^{3,4}$ Según el mecanismo del daño inmunológico involucrado, las RAM se agrupan de acuerdo con la clasificación de Gell y Coombs:,5
- Reacciones tipo I, mediadas por IgE, que se desencadenan pocos minutos después de la segunda administración de un fármaco; pueden ocasionar anafilaxia y muerte.

- Reacciones tipo II, debidas a citotoxicidad mediada por anticuerpos y relacionadas con cuadros clínicos graves, con participación de IgG e IgM que inducen daño celular como citopenias, lupus like, etcétera.

- Reacciones tipo III que se presentan por la formación de complejos inmunes que pueden depositarse en glomérulos, piel, plexos, etcétera, causando daño como vasculitis, pleuritis, glomerulonefritis, exantema y artralgias, con duración de una a tres semanas.

- Reacciones tipo $I V$, tardías o retardadas, ocurren después de 72 horas, son mediadas por linfocitos T, macrófagos, NK y linfocitos citotóxicos. Debido a 
la cantidad de células implicadas hay cuatro subcategorías según el tipo de células predominantes: tipo IVa o Th1, con participación de INF- $\gamma$, TNF- $\alpha$ y activación de macrófagos; tipo IVb o Th2, con activación de eosinófilos y participación de citocinas IL-4, IL-5 e IL-13; tipo IVc por LT citotóxicos y tipo IVd o neutrofílica ${ }^{3,4,7,8}$ (Cuadro 1).

Los factores de riesgo para desarrollar hipersensibilidad a fármacos son el sexo femenino, la regu- lación inmunogénica, las edades extremas, así como las enfermedades virales, hepáticas y autoinmunes; la atopia condiciona mayor severidad.

Los fármacos o sus metabolitos pueden reaccionar directamente con las proteínas y la superficie celular, o mediante procesos metabólicos transformarse en moléculas con mayor polaridad e hidrofilia, capaces de unirse a macromoléculas, principalmente proteínas endógenas (albúmina y globulinas séricas). Cualquiera que sea la estructura funcional

\begin{tabular}{|c|c|c|c|c|}
\hline Tipo & Mediado por & Clínica & Diagnóstico in vitro & Diagnóstico in vivo \\
\hline 1 & $\begin{array}{l}\text { Mediada por lgE } \\
\text { Eosinófilos, mastoci- } \\
\text { tos y basófilos } \\
\text { (inmediata) }\end{array}$ & $\begin{array}{l}\text { Urticaria } \\
\text { Angioedema } \\
\text { Rinitis } \\
\text { Broncoespasmo } \\
\text { Anafilaxia }\end{array}$ & $\begin{array}{l}\text { IgE específica } \\
\text { Triptasa sérica } \\
\text { Prueba de estimulación celular } \\
\text { (CAST) } \\
\text { Técnica de activación de basófilos } \\
\text { (TAB) (DB, CD63) }\end{array}$ & $\begin{array}{l}\text { Pruebas cutáneas } \\
\text { (prick, intradérmicas) } \\
\text { Prueba de reto }\end{array}$ \\
\hline II & $\begin{array}{l}\text { Citotoxicidad } \\
\text { dependiente de } \\
\text { anticuerpos lgG e } \\
\text { lgM (mediata) }\end{array}$ & $\begin{array}{l}\text { Anemia hemolítica } \\
\text { Trombocitopenia } \\
\text { Neutropenia } \\
\text { Autoinmunidad }\end{array}$ & $\begin{array}{l}\text { Test de Coombs } \\
\text { Anticuerpos versus plaquetas } \\
\text { Anticuerpos versus neutrófilos }\end{array}$ & $\begin{array}{l}\text { Solo los retos al fár- } \\
\text { maco pueden hacer el } \\
\text { diagnóstico, pero son } \\
\text { de alto riesgo }\end{array}$ \\
\hline III & $\begin{array}{l}\text { Por depósito de } \\
\text { inmunocomplejos } \\
\text { (IgG e IgM) } \\
\text { (mediata) }\end{array}$ & $\begin{array}{l}\text { Enfermedad del suero } \\
\text { Vasculitis } \\
\text { Glomerulonefritis } \\
\text { Fiebre medicamentosa }\end{array}$ & $\begin{array}{l}\text { C3, C4, ANA, ANCA, CCP, antitiroi- } \\
\text { deos, etc. } \\
\text { Pruebas de función hepática y renal } \\
\text { Anatomía patológica }\end{array}$ & $\begin{array}{l}\text { Biopsias con inmu- } \\
\text { nofluorescencia }\end{array}$ \\
\hline IVa & TH1 & $\begin{array}{l}\text { Dermatitis por } \\
\text { contacto }\end{array}$ & $\begin{array}{l}\text { Test de transformación linfocita- } \\
\text { ria (TTL), técnica modificada de } \\
\text { factor inhibidor de la migración } \\
\text { de leucocitos (LIF), precursores } \\
\text { de linfocitos T citotóxicos (CTLp), } \\
\text { citocinas }\end{array}$ & Pruebas de parche \\
\hline IVb & $\mathrm{TH} 2$ & $\begin{array}{l}\text { Exantemas maculopa- } \\
\text { pulares con eosinofilia } \\
\text { (DRESS) }\end{array}$ & $\begin{array}{l}\text { BHC con celularidad para buscar } \\
\text { eosinófilos. } \\
\text { Linfocitos atípicos, TTL, LIF }\end{array}$ & Pruebas de parche \\
\hline IVc & LTC & $\begin{array}{l}\text { Dermatitis de contacto, } \\
\text { enfermedades macu- } \\
\text { lopapulares y bullosas } \\
\text { (Síndrome de Steven } \\
\text { Johnson, NET) }\end{array}$ & $\begin{array}{l}\text { LIF } \\
\text { Pruebas de función hepática } \\
\text { Actividad de IgM versus virus her- } \\
\text { pes, Epstein Barr y citomegalovirus }\end{array}$ & Pruebas de parche \\
\hline IVd & Neutrófilos & $\begin{array}{l}\text { AGEP (pustulosis exán- } \\
\text { tematica aguda genera- } \\
\text { lizada), farmacodermias } \\
\text { asociadas a neutrofilia }\end{array}$ & $\mathrm{BHC}$ & Pruebas de parche \\
\hline
\end{tabular}




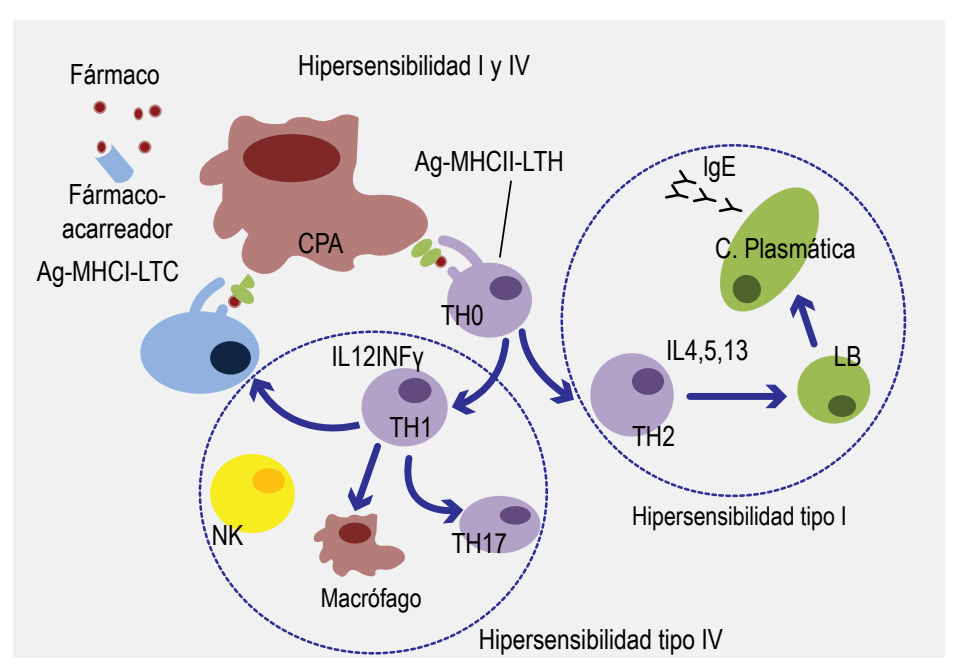

Figura 1. Esquema de la respuesta inmunológica a los fármacos. El fármaco, hapteno o sus metabolitos pueden reaccionar directamente con proteínas para ser procesados y presentados como antígenos en las CPA en el contexto MHC-TCR para activar linfocitos THO, desencadenándose la polarización y la producción final de efectores celulares (Th1) y humorales (Th2) de la hipersensibilidad tipos IV y I, respectivamente.

que tome el fármaco unido a las proteínas acarreadoras, provoca el desarrollo de hipersensibilidad ${ }^{7-9}$ (Figura 1).

Existen tres tipos de fármacos y vías de sensibilización de la respuesta inmunológica en la alergia a medicamentos.

- Fármacos químicamente activos procesados por las células presentadoras de antígenos (CPA).

- Fármacos no activos que después de su metabolismo hepático producen metabolitos que se convierten en prohaptenos aptos para su procesamiento y presentación.

- Fármacos inertes que activan ciertos receptores de células T (TCR). ${ }^{9,10}$

El diagnóstico de alergia a medicamentos se basa en 3 aspectos: historia clínica, pruebas in vitro e in vivo y pruebas de provocación (estándar de oro).

Se dispone de pocas pruebas in vitro para el diagnóstico de hipersensibilidad:

- Para la hipersensibilidad tipo I, la degranulación de basófilos (DB) mediante la prueba de Shelley, Morton y Soifer, así como otras pruebas mediadas por IgE (RAST, INMUNO CAP, CAST) para algunos fármacos como penicilina, determinación de histamina plasmática, triptasa y la activación de basófilos (TAB-CD63).

- Para la hipersensibilidad tipo IV, TTL, LIF (leukocyte migration inhibiton factor), producción de citocinas y ensayos de citotoxicidad. ${ }^{4,7,11-14}$

\section{Objetivos}

Realizar pruebas modificadas de DB y LIF en pacientes con diagnóstico de hipersensibilidad a medicamentos, para evaluar la confiabilidad y complementariedad de ambas pruebas, así como la utilidad de manejar diluciones para mejorar la sensibilidad.

\section{Métodos}

Se incluyeron pacientes de uno y otro sexo, sin límite de edad, ambulatorios y hospitalizados, residentes en la Ciudad de México, enviados a un laboratorio para realización de pruebas modificadas de DB, LIF o ambas, con diagnóstico de hipersensibilidad a medicamentos de acuerdo con su historia clínica. Se registraron datos demográficos y síntomas predominantes. Las pruebas se realizaron entre diciembre de 2009 y diciembre de 2014.

Las pruebas para DB (hipersensibilidad tipo I) y LIF (hipersensibilidad tipo IV) fueron modificadas con estandarizaciones previas (curvas dosis y tiempo de respuesta), concentración de basófilos (3 a 5 veces), tinción de los basófilos (azul de toluidina, Wright y rojo de metilo), uso de controles químicos (positivo-formil-metil-leucin-fenilalanina [f-MLP]2 mM, negativo o basal), solución salina fisiológica y valores de referencia obtenidos de población no alérgica $(\mathrm{n}=26)$.

Para la prueba modificada de DB se tomaron $20 \mathrm{~mL}$ de sangre venosa (heparina $158 \mathrm{USP}-10 \mathrm{~mL}$ ) extraídos con un sistema de venopunción (Vacutainer®, Becton, Dickinson). La suspensión de basófilos concentrada se incubó con el fármaco por retar 
o en sospecha, a concentraciones de $1 \mathrm{mg} / \mathrm{mL}$ y 0.1 $\mathrm{mg} / \mathrm{mL}$, con controles químicos negativo y positivo durante 20 minutos, a $37^{\circ} \mathrm{C}$. Se hicieron frotis por duplicado, se tiñeron y en el microscopio se realizó un conteo por cada 2000 células; para calcular el porcentaje de degranulación se comparó el número de basófilos con el de la muestra control basal.

Para el LIF se requirió también $20 \mathrm{~mL}$ de sangre venosa, así como concentración y lavado de los leucocitos con RPMI. Los microhematócritos se cortaron e incubaron en cámaras de Bloom con un control negativo y con un positivo, así como con $1 \mathrm{mg} / \mathrm{mL}$ y $0.1 \mathrm{mg} / \mathrm{mL}$ del fármaco, a $37^{\circ} \mathrm{C}$. Las muestras fueron observadas a las 2, 4, 6 y 18 horas, para medir las áreas de migración de leucocitos y realizar el cálculo (porcentaje de LIF) en comparación con las muestras testigo negativas.

El valor de referencia para la prueba modificada de DB es 0 a $30 \%$ de degranulación y para LIF, 0 a $25 \%$ de inhibición de la migración de leucocitos.

Los resultados fueron analizados mediante correlación bivariada de Kendall y Spearman y estadística descriptiva de frecuencias, medidas de asociación y pruebas de especificidad y especificidad.

\section{Resultados}

Se estudiaron 177 pacientes de diciembre 2009 a diciembre 2014, distribuidos en grupos etarios; 115 (64.97\%) requirieron estudios de 1 a 3 medicamentos, $53(29.94 \%)$ de 4 a 6 medicamentos y $9(5.08 \%)$ de 7 a 18 fármacos. A 125 pacientes se solicitó solo prueba modificada de $\mathrm{DB}$, a 51 ambas pruebas y a un paciente solo de LIF, para realizar un total de 762 pruebas: $561(73.62 \%)$ de DB y $201(26.37 \%)$ de LIF. Los pacientes incluidos en el estudio tuvieron RAM con o sin antecedentes de exposición previa al fármaco, antecedentes familiares de atopia, así como síntomas y reacciones de hipersensibilidad inmediata o tardía. No se realizaron pruebas de provocación.

De los 177 pacientes, 170 (96.04\%) fueron positivos a cuando menos un medicamento por alguna de las dos pruebas (RR de 4.71). Los estudios se realizaron con mayor frecuencia en mujeres $(114,64.41 \%)$, cuya edad media fue de 38.5 años, predominio que se observó en todos los grupos etarios, excepto en el grupo de 11 a 20 años, en el que aumentaron los hombres (edad media de 13 años). El grupo etario más frecuente fue el de 41 a 50 años y el de mayor solicitud de estudios, 7 a 18 medicamentos ( 40 a $50 \%$ positivos), correspondió al de 51 a 60 años (Figura 2).

Como la prueba modificada de DB se considera positiva con valores mayores a $30 \%$ de degranulación y la de LIF con valores mayores a $25 \%$ de inhibición, 394 pruebas $(70.23 \%)$ de DB fueron positivas y 167 fueron negativas. Respecto a las pruebas control de población no alérgica, 26 fueron negativas y 0 positivas. La historia clínica permitió calcular la sensibilidad de la prueba modificada de DB en 0.845 (84\%), especificidad de $100 \%$, con coeficiente de variación de $10 \%$. El número de basófilos promedio fue de 19.9 en 2000 células $\left(250 \times 10^{3} \mu \mathrm{L}\right)$, con un rango de 7 a 43 ; la respuesta para el control positivo con f-MLP tuvo un promedio de $56.16 \%$ y un rango de 25 a $94 \%$ de degranulación, más alto que en la población no alér-

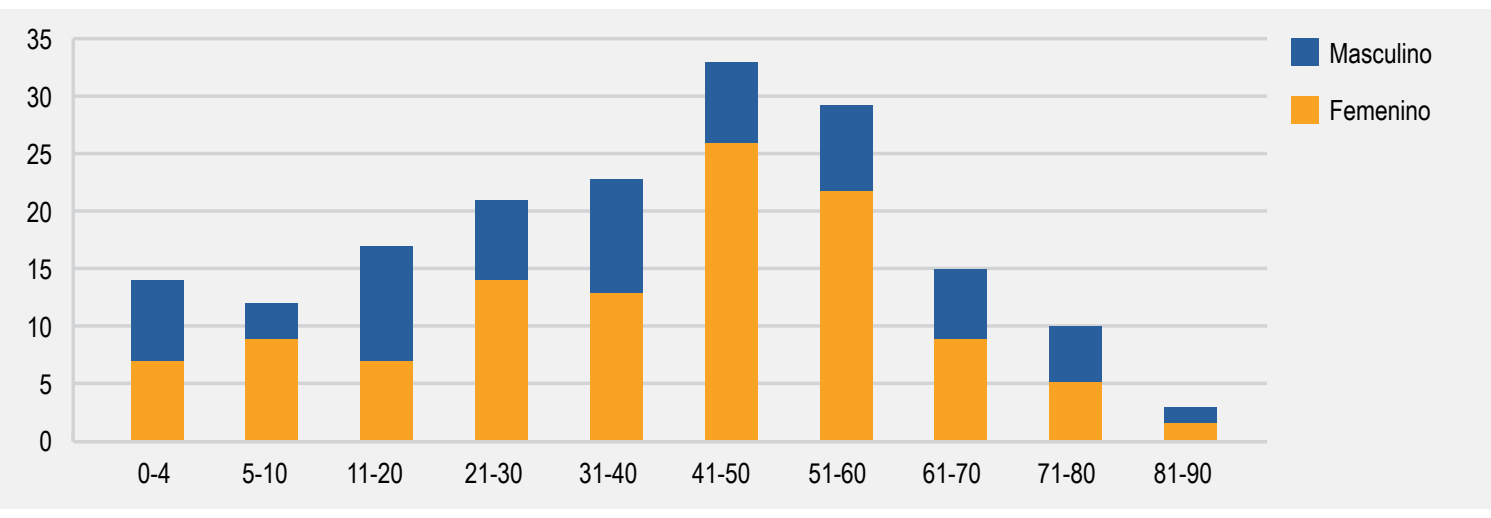

Figura 2. Distribución por sexo de los pacientes con sospecha de alergia a medicamentos enviados a pruebas en un laboratorio, 2009 a 2014. 


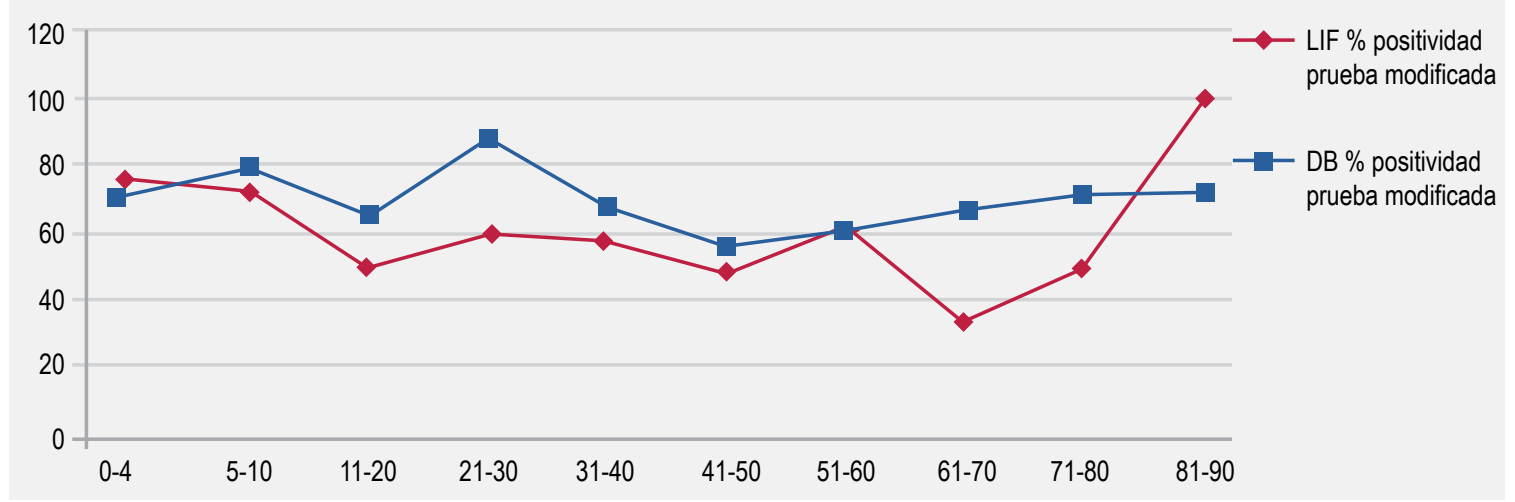

Figura 3. Positividad en las pruebas modificadas de LIF y DB según los grupos de edad de población con alergia a medicamentos.

gica; para el control negativo no hubo diferencias significativas.

De 201 pruebas modificadas de LIF realizadas, 135 fueron positivas $(67.16 \%)$ y 66 negativas; de las pruebas control de referencia, 26 fueron negativas y 0 positivas, lo que permitió calcular la sensibilidad en $0.775(77 \%)$ y la especificidad en $100 \%$, con un coeficiente de variación de $12 \%$.

Al realizar las pruebas modificadas de DB y LIF en forma complementaria y a dos concentraciones
( $1 \mathrm{mg} / \mathrm{mL}$ y $0.1 \mathrm{mg} / \mathrm{mL}$ ), se encontró que su positividad, sensibilidad y utilidad se incrementaron $20 \%$.

El análisis de correlación de las pruebas modificadas de DB y LIF se realizó con los resultados del grupo al que se le solicitó ambas ( $\mathrm{n}=51)$. Mediante la prueba de correlación de Pearson con $1 \mathrm{mg} / \mathrm{mL}$ se obtuvo $0.396(\mathrm{p}=0.0001)$; mediante el coeficiente de Spearman, $0.392(\mathrm{p}=0.0001)$ y con el coeficiente de Kendall, $0.273(\mathrm{p}=0.01)$. Estos resultados demuestran una correlación significativa a nivel bilateral de

\begin{tabular}{|c|c|c|c|c|c|c|c|}
\hline Fármacos & $\begin{array}{l}\text { Número de } \\
\text { fármacos }\end{array}$ & $\begin{array}{l}\text { \% Fármacos } \\
\text { solicitados }\end{array}$ & $\begin{array}{c}\text { LIF* }^{*} \\
\text { solicitadas }\end{array}$ & $\begin{array}{c}\% \text { LIF* }^{*} \\
\text { positivas }\end{array}$ & $\begin{array}{c}\mathrm{DB}^{*} \\
\text { solicitadas }\end{array}$ & $\begin{array}{c}\% \mathrm{DB}^{*} \\
\text { positivas }\end{array}$ & $\begin{array}{c}\% \text { positivas } \\
\text { global }\end{array}$ \\
\hline Antibióticos & 217 & 37.87 & 70 & 58.57 & 211 & 72.09 & 71.4 \\
\hline $\begin{array}{l}\text { Antinflamatorios: } \\
\text { AINE y esteroi- } \\
\text { deos }\end{array}$ & 129 & 22.51 & 48 & 68.57 & 127 & 62.20 & 74.4 \\
\hline Anestésicos & 48 & 8.37 & 21 & 38.09 & 48 & 60.41 & 62.5 \\
\hline Analgésicos & 39 & 6.80 & 8 & 50 & 39 & 66.66 & 66.7 \\
\hline $\begin{array}{l}\text { Suplementos } \\
\text { alimenticios y } \\
\text { vitaminas }\end{array}$ & 22 & 3.84 & 10 & 40 & 22 & 59.09 & 72.7 \\
\hline Antihipertensivos & 15 & 2.62 & 7 & 85.7 & 15 & 80.00 & 86.66 \\
\hline Ansiolíticos & 13 & 2.27 & 6 & 50 & 13 & 38.94 & 53.8 \\
\hline Hormonas & 10 & 1.74 & 6 & 33.33 & 10 & 50.00 & 50.0 \\
\hline Antiretrovirales & 9 & 1.57 & 1 & 0 & 9 & 66.66 & 66.66 \\
\hline Otros & 71 & 12.39 & 22 & 68.18 & 69 & 56.71 & 60.6 \\
\hline Total & 573 & 99.98 & 199 & $49.24 \%$ & 563 & 61.27 & 66.5 \\
\hline
\end{tabular}

LIF, factor de inhibición de la migración de leucocitos; DB, degranulación de basófilos; *Pruebas modificadas 


\begin{tabular}{|l|c|c|c|c|c|}
\hline \multicolumn{2}{l}{ Cuadro 3. Antibióticos con los que se realizaron pruebas modificadas de LIF y DB } & & \\
\hline Antibióticos & $\begin{array}{c}\text { Número de } \\
\text { pruebas }\end{array}$ & $\begin{array}{c}\text { LIF* }^{*} \\
\text { solicitadas }\end{array}$ & $\begin{array}{c}\% \text { LIF* } \\
\text { positivas }\end{array}$ & $\begin{array}{c}\text { DB }^{*} \\
\text { solicitadas }\end{array}$ & $\begin{array}{c}\%_{\text {DB }}^{*} \\
\text { positivas }\end{array}$ \\
\hline Penicilina & 21 & 10 & 100 & 21 & 95.23 \\
\hline Betalactámicos & 26 & 8 & 62.5 & 18 & 73.08 \\
\hline Cefalosporinas & 30 & 13 & 30.76 & 28 & 71.43 \\
\hline Sulfas & 32 & 7 & 71.42 & 31 & 80.64 \\
\hline Fluoroquinolonas & 27 & 10 & 70 & 17 & 68 \\
\hline Macrólidos & 23 & 7 & 57.14 & 23 & 69.56 \\
\hline
\end{tabular}

LIF, factor inhibidor de la migración de leucocitos; DB, degranulación de basófilos; *Pruebas modificadas

0.01. La correlación de las pruebas modificadas de DB y LIF a concentración de $0.1 \mathrm{mg} / \mathrm{mL}$ mediante la prueba de correlación de Pearson fue de $0.310(\mathrm{p}=0.0001)$; mediante el coeficiente de Spearman fue de 0.260 $(p=0.002)$ y con el coeficiente de Kendall, de 0.26 $(\mathrm{p}=0.002)$. Lo que traduce una correlación significativa a nivel bilateral de 0.01 .

La positividad promedio de DB fue mayor en comparación con el LIF en todos los grupos etarios, excepto en el grupo geriátrico, en el cual LIF fue mayor (Figura 3).

Se realizaron 762 pruebas de los medicamentos, los cuales fueron divididos en 10 grupos: antibióticos, antiinflamatorios, anestésicos, analgésicos, suplementos alimenticios y vitaminas, antihipertensivos, ansiolíticos, hormonas, antirretrovirales y otros (antilipídicos, antiácidos, antineoplásicos, antiurémicos, antidepresivos, medicamentos para el control de peso, entre otros) (Cuadro 2).

Se realizó el estudio por separado de los fármacos más representativos que integran los diferentes grupos de fármacos: antibióticos y antiinflamatorios no esteroideos (Cuadros 3 y 4).
La penicilina tuvo la mayor respuesta positiva tanto en LIF como en DB, seguida de las sulfas, derivados de betalactámicos, fluoroquinolonas y macrólidos. Por su parte, el diclofenaco presentó los resultados más altos en las pruebas modificadas de LIF y DB, seguido del naproxeno y el ibuprofeno; el menos reactivo fue el ácido acetilsalicílico.

\section{Discusión}

La mayoría de los resultados de las pruebas modificadas de DB y LIF solicitadas con base en la historia clínica correlacionaron con los síntomas de los pacientes cuando tomaron los medicamentos; 96.04\% de los 177 pacientes fueron positivos por lo menos a un medicamento por alguna de las dos pruebas, lo que sugiere congruencia diagnóstica debido a que la sensibilidad y especificidad de las pruebas fueron buenas.

Los resultados se calcularon con base en la historia clínica sin considerar las pruebas de provocación, debido a la fuerte relación del síntoma con el fármaco implicado, lo que constituye un reto natural. En la mayoría de los pacientes se solicitó pruebas a más de un medicamento debido a que la reacción se presentó

\begin{tabular}{|c|c|c|c|c|c|}
\hline AINE & $\begin{array}{c}\text { Número } \\
\text { de pruebas }\end{array}$ & $\begin{array}{c}\text { LIF* }^{*} \\
\text { solicitadas }\end{array}$ & $\begin{array}{l}\text { \% LIF* } \\
\text { positivas }\end{array}$ & $\begin{array}{c}\mathrm{DB}^{*} \\
\text { solicitadas }\end{array}$ & $\begin{array}{c}\% \mathrm{DB}^{*} \\
\text { positivas }\end{array}$ \\
\hline Diclofenaco & 15 & 8 & 100 & 14 & 78.6 \\
\hline Naproxeno & 23 & 10 & 70 & 23 & 56.52 \\
\hline Ácido acetilsalicílico & 17 & 5 & 0 & 17 & 52.94 \\
\hline Ibuprofeno & 18 & 6 & 37.5 & 18 & 68.75 \\
\hline
\end{tabular}


mientras eran tratados con varios fármacos, lo cual se justifica por la mayor prevalencia de pacientes con edad entre los 50 y 70 años y el abuso de AINE.

La DB fue la prueba más solicitada $(74 \%)$, seguida del LIF (26\%), lo que coincide con las manifestaciones clínicas más frecuentemente mediadas por liberación de histamina y solo ocasionalmente por vía celular: urticarias $(66.66 \%)$, anafilaxia $(12.5 \%)$ y angioedema $(33.33 \%)$. Se solicitaron ambas pruebas en pacientes con reacciones maculopapulares de síndrome de Steven-Johnson y síndrome DRESS.4-7,10,11

El predominio del sexo femenino ha sido señalado por otros autores y manifiesta una posible interrelación entre el sistema endocrino y el sistema inmunológico debido al efecto de los estrógenos, que aumentan la expresión de moléculas $\mathrm{MHC}$ y la liberación de citocinas (IL-4, IL-15 e IL-13), que favorecen la polarización a Th2 con la consiguiente producción de anticuerpos isotipo IgE, característicos de la respuesta de hipersensibilidad tipo I. ${ }^{5,6,15,16}$

Los grupos etarios de 41 a 50 años y de 51 a 60 años fueron los más frecuentes con aumento de la reactividad múltiple, lo que ha sido descrito como resultado del uso crónico de múltiples fármacos, disfunción hepática, estrés, enfermedades infecciosas y metabólicas típicas de la población mexicana. ${ }^{17}$

$\mathrm{El}$ análisis de correlación de las dos pruebas modificas se realizó con los resultados de 190 pruebas de LIF contra DB y a 2 concentraciones: $1 \mathrm{mg} / \mathrm{mL}$ y $0.1 \mathrm{mg} / \mathrm{mL}$, con pruebas estadísticas paramétricas y no paramétricas (Pearson, Spearman y Kendall), obteniendo una correlación positiva con alta significación a nivel bilateral de 0.001. Estos resultados indican que hay una asociación positiva entre ambas pruebas, lo que pone de manifiesto que los medicamentos pueden activar la respuesta inmune polarizándola tanto por uno como por otro mecanismo., ${ }^{718}$

La frecuencia y positividad a medicamentos identificadas en este estudio concuerdan con la distribución reportada en la bibliografía médica inter- nacional: antibióticos, antiinflamatorios, anestésicos, analgésicos, antihipertensivos. Los antibióticos más solicitados en todos los grupos etarios fueron la penicilina y sus derivados, ya que aún constituyen la primera línea de tratamiento seguidos de sulfas, fluoroquinolonas y macrólidos. Las penicilinas fueron los fármacos que mostraron más reactividad en las dos pruebas, sin embargo, cabe mencionar que las cefalosporinas indujeron más respuestas tipo I que IV. Las sulfas dieron resultados positivos para ambas pruebas, al igual que las fluoroquinolonas y los macrólidos, incluidos debido a que había pacientes con reacciones maculopapulares por estos fármacos. ${ }^{2,3,5,6}$

El segundo grupo fue el de los AINE, que debido a su estructura química reactiva (aromáticos del benceno, heterocíclicos de nitrógeno y aril acéticos) favorecen la unión a proteínas acarreadoras, estimulación del sistema inmune con polarización a la hipersensibilidad tipo I y solo en algunos casos de tipo IV.

Sería importante hacer el análisis biomolecular básico para comprender los cruces antigénicos y el tipo de respuesta inmune aún no desarrollada, lo que posibilitaría tomar las medidas necesarias para un mejor diagnóstico, prevención y tratamiento.

\section{Conclusiones}

Las reacciones alérgicas a fármacos son más frecuentes en mujeres en la cuarta y quinta décadas de la vida. La prueba modificada de DB es útil para el diagnóstico de hipersensibilidad tipo I, el mecanismo más frecuentemente implicado. Llama la atención que los ancianos tuvieron más respuestas de tipo tardía o celular, que los convierte en pacientes de riesgo para farmacodermias graves tipo IV como síndromes de Steven-Johnson, DRESS y DISH.

La sensibilidad y utilidad de ambas pruebas mejoran al realizarlas con diluciones y en forma complementaria, con lo que se apoya el diagnóstico, la prevención y el tratamiento.

\section{Referencias}

1. Dreser A, Wirtz VJ, Corbett KK, Echaniz G. Uso de antibióticos en México: revisión de problemas y políticas. Salud Publica Mex. 2008;50 supl 4:480-487.

2. Padilla SMT, Arias CA, Macías WA, González DSN, Galindo RG, García CCY. Prevalencia de alergia a medicamentos en un grupo de niños y adolescentes asmáticos del noreste de México. Rev Alerg Mex. 2006;53(5):179-182. 
3. Bolte C. Reacciones medicamentosas severas en piel. Rev Med Clin Condes. 2011;22(6):757-65. doi: 10.1016/S0716-8640(11)70488-6

4. Giner M. Alergia a medicamentos. Conceptos básicos y actitud a seguir por el pediatra. Protoc Diagn Ter Pediatr. 2013;1:1-24.

5. Giner-Muñoz MT. Hipersensibilidad a medicamentos. Pediatr Integral. 2009;13:819-834.

6. Becerril-Ángeles M, Aranda-Jan A, Moreno-Quiroz J. Encuesta de reacciones adversas a medicamentos en pacientes hospitalizados. Rev Alerg Mex. 2011;58(4):179-184.

7. Mayorga C, Sanz ML, Gamboa P, García-Avilés MC, Fernández J, Torres MJ, et al. In vitro methods for diagnosing nonimmediate hypersensitivity reactions to drugs. J Allergy Clin Immunol. 2013;23(4):213225.

8. Karakaya G, Isik SR, Kalyouncu AF. Determining safe antibiotics for dug hypersensitive patients with the alternative method of double-triple test. Allergol Immunopathol (Madr). 2008;36:264-270.

9. Pichler WJ. Delayed drug hypersensitivity reactions. Ann Intern Med. 2003;139(8):683-693.

10. Vilella-Puig R. La respuesta inmunoalérgica mediada por IgE. Arch Bronconeumol. 2006;42 supl 1:6-12.

11. Mora NA, Segura MNH, Almeida AV, Díaz TMT, Martínez PT. Alergia a medicamentos evaluada con la prueba de Shelley modificada. Serie de casos. Rev Alerg Mex. 2006;53(3):89-93.

12. Rangel $\mathrm{CH}$, Montero-Mora $\mathrm{P}$, Espinosa-Larrañaga $\mathrm{F}$, Castillo-V FJ. Factor inhibidor de la migración de leucocitos y degranulación de basófilos en las reacciones a medicamento. Rev Alerg Mex. 1991;38(4):105-109.

13. Sanz ML, García-Avilés MC, Gamboa PM. Producción antígeno-específica de sulfidoleucotrienos mediante CAST (cellular assay stimulation test) en el diagnóstico alergológico in vitro. Arch Alerg Inmunol Clin. 2006;37:12-33.

14. Sanz-Larruga MI, García MC, Caballero MR, Diéguez I, Gamboa PM. Test de activación de basófilos en el diagnóstico de alergia a medicamentos. An Sis San Navarra. 2003;26 supl 2:39-47. Disponible en: http://recyt.fecyt.es/index.php/ASSN/article/view/4987

15. De León-Nava M, Morales-Montor J. Dimorfismo sexual inmunitario: ¿pueden los esteroides sexuales polarizar el perfil de citocinas Th1/Th? Rev Invest Clin. 2006;58(2):161-169.

16. Barañao RI. Hormonas sexuales y respuesta inmunológica. SAEGRE. 2009;16(2):20-30. Disponible en: http://ttvps.com/saegre/revista/numeros/2009/n2/revision_n2.pdf

17. Novak N, Kraft S. Receptores para IgE. Curr Opin Immunol. 2001;13:721-726.

18. Rocklin RE. Mediators of cellular immunity, their nature and assay. J Invest Dermatol. 1976;67(3):372379. 